\title{
Comparison of Sesamoid Bone Position and Hallux Valgus Angle in Weight Bearing Conditions between Subjects with and without Hallux Valgus
}

\author{
Moon-Hwan Kim', In-Cheol Jeon², Ui-Jae Hwang'ㄹ, Young Kim³ \\ 'Department of Rehabilitation Medicine, Wonju Severance Christian Hospital, Wonju; ${ }^{2}$ Department of Physical Therapy, The Graduate School, Yonsei \\ University, Wonju; ${ }^{3}$ Department of ICT Convergence Rehabilitation Engineering, Soonchunhyang University, Asan, Korea
}

Purpose: Previous studies reported changes in the first metatarsophalangeal (MTP) joint angle in relation with and without weight bearing, but it is unclear whether sesamoid bone of the great toe changes in weight bearing conditions particularly in subjects with hallux valgus (HV). To investigate how weight bearing conditions can affect the position of the medial sesamoid bone (MSB), first MTP joint angle, and second intermetatarsal angle (IMA) in the recruited subjects.

Methods: Subjects were recruited 24 with HV and 21 without HV in study. X-rays were taken in the weight bearing and non-weight bearing conditions. The distance of the MSB, first MTP joint angle, and second IMA were measured from the radiographs. Data were analyzed by paired and Independent t-test. The statistical significance level was $p<0.05$.

Results: In both groups, the first MTP joint angles and the distance of the MSB were significantly smaller, while the second IMA was significantly greater in the weight bearing condition. The difference in the distance of the MSB between the two postures was significantly greater in the group with HV.

Conclusion: Weight bearing can affect the first MTP joint angle, second IMA, and position of the MSB; the change in the position of the MSB in weight bearing was greater in the group with hallux valgus. The difference in these variables between weight bearing and nonweight bearing conditions may be considered when measuring HV.

Keywords: Hallux valgus, Radiography, Sesamoid bones

\section{INTRODUCTION}

A sesamoid bone is a small oval-shaped bone measuring 5-10 $\mathrm{mm}$ in length. ${ }^{1}$ There are two located directly below the metatarsal of the great toe., ${ }^{1,2}$ The mobility of sesamoid bones is an important component of the mechanics of the first metatarsophalangeal (MTP) joint, ${ }^{3}$ as the main function of the sesamoid bones is to reduce friction between tendons and surrounding rigid structures, and to facilitate gliding movements between adjacent tissues. ${ }^{4}$ The medial and lateral sesamoid bones are generally displaced up to 10$12 \mathrm{~mm}$ when the first MTP joint is flexed and these changes in turn interfere with the normal mechanisms of the first MTP joint. ${ }^{5}$

Commonly, the sesamoid bones are displaced in individuals with

Received Nov 18, 2016 Revised Dec 11, 2016

Accepted Dec 12, 2016

Corresponding author Young Kim

E-mail saleeva@naver.com hallux valgus (HV), which is defined as a progressive deformity involving lateral deviation of the great toe at the MTP joint that can result in a painful prominence of the medial aspect of the first metatarsal head. ${ }^{6,7}$ The HV was shown high incidence over forty adults. ${ }^{8}$ The severity of HV is classified according to the lateral deviation of the first MTP as normal $\left(\leq 15^{\circ}\right)$, mild $\left(<20^{\circ}\right)$, moderate $\left(20-40^{\circ}\right)$, and severe $\left(>40^{\circ}\right){ }^{9} \mathrm{HV}$ is characterized by lateral subluxation of the flexor hallucis longus muscle, flexor hallucis brevis, and sesamoid bones. ${ }^{3,5}$ Laterally deviated sesamoid bones lead to reduced plantar flexion of the first MTP joint, because the flexor hallucis longus tendon passes between the sesamoid bones, and the flexor hallucis brevis muscles that extend onto the sesamoid bones serve as adductors rather than flexors of the great toe., ${ }^{5,10,11}$ Consequently, the posi-

Copylight (C2016 The Korea Society of Physical Therapy

This is an Open Access article distribute under the terms of the Creative Commons Attribution Non-commercial License (Http:// creativecommons.org/license/by-nc/4.o.) which permits unrestricted non-commercial use, distribution, and reproduction in any medium, provided the original work is properly cited. 
tion of the sesamoid bones is an important determinant of function of the first ray and thus need to be considered when measuring $\mathrm{HV}^{12}$

Many studies have investigated changes in HV based on radiographic assessment. ${ }^{13-15}$ The HV angle and the 2nd to the 5th MTP joint angles differ in $\mathrm{HV}$ patients in weight bearing condition when compared with non-weight bearing. ${ }^{16,17}$ Although the position of the sesamoid bones play an important role in biomechanics of the first MTP joint, no study has investigated the difference in position changes of the medial sesamoid bone (MSB) between weight bearing and non-weight bearing conditions in subjects with HV. Confirming the position of the sesamoid bone, which reflects the surrounding soft tissues in weight bearing and non-weight bearing, can later suggest proper posture for hallux valgus exercise. Therefore, this study compared the position of the MSB, the HV angle, and the second intermetatarsal angle (IMA) between weight bearing and non-weight bearing groups in subjects with HV.

\section{METHODS}

\section{Subjects}

A total of 45 subjects were included and divided 2 groups (HV group and non-HV group). 24 subjects who were diagnosed with HV (13 men, 11 women) with a mean age of $22.49 \pm 2.40$ years, mean height of $169.10 \pm 8.62 \mathrm{~cm}$, and mean weight of $63.38 \pm 12.31 \mathrm{~kg}$ (HV group) and 21 healthy individuals without HV (11 men, 10 women) with a mean age of $21.77 \pm 2.17$ years, height of $169.71 \pm 7.34 \mathrm{~cm}$, and weight of 60.58 $\pm 9.91 \mathrm{~kg}$ (non-HV group) were recruited for this study. The HV group comprised 39 feet with a mean first MTP joint angle of $18.88 \pm 3.06^{\circ}$, and the non-HV group had 31 feet with a mean first MTP joint angle of $10.34 \pm 3.30^{\circ}$ when measured in standing (weight bearing condition). Subjects were excluded from the study if they had any neuromuscular disorder, history of foot and ankle surgery, rheumatoid arthritis, neuropathic diabetes, or tumors. All subjects were informed about the study methods and volunteered to participate.

\section{Experimental methods}

All radiographs used in this study were taken by a radiology technologist at an orthopedic clinic using a diagnostic table and X-ray machine (D Company). The foot was X-rayed in standing position and while sitting on a chair with the knees flexed to $90^{\circ}$. All of the

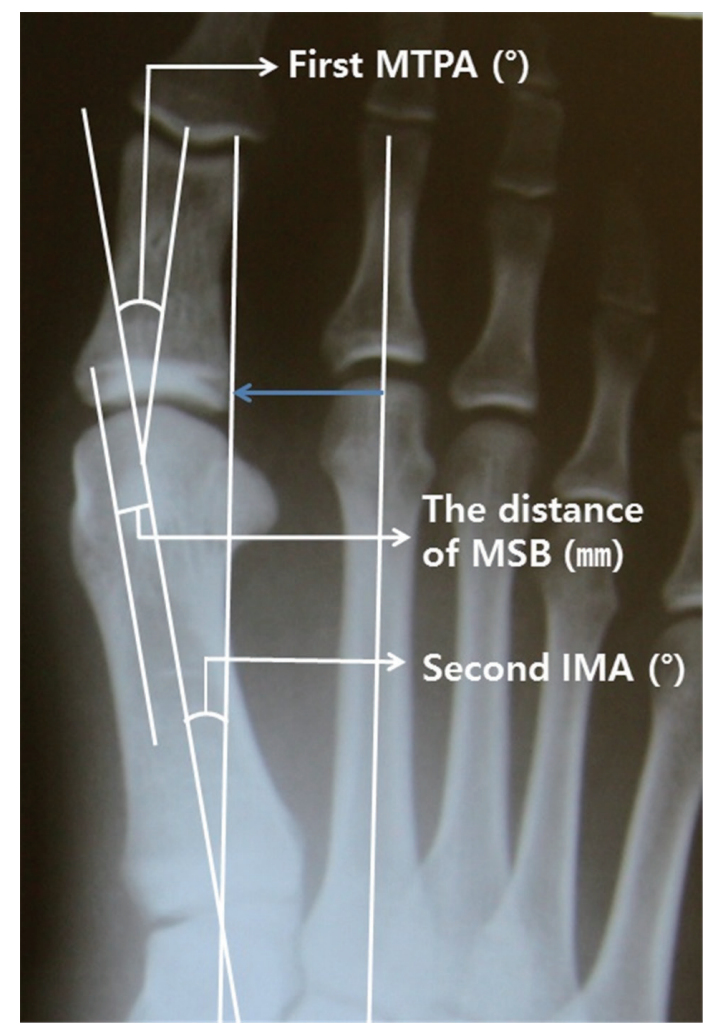

Figure 1. Measurement of the distance of MSB, first MTPA, and second IMA angle.

MSB: medial sesamoid bone, MTPA: metatarsophalangeal joint angle, IMA: intermetatarsal angle.

feet were X-rayed in the dorsoplantar direction. Radiographs of the foot taken in the two postures were used to measure the position of the MSB (vertical distance between the distal medial edge of the MSB and longitudinal axis of the first metatarsal bone), the first MTP joint angle (the angle created by the longitudinal axes of the first metatarsal and proximal phalange), and the second IMA (the angle created by the longitudinal axes of the first and second metatarsals) using Centricity PACS RA1000 workstation (ver. 2.1.0, GE Healthcare Integrated IT Solutions, Barrington, USA) (Figure 1). The longitudinal axes of the first and second metatarsals were indicated by the commonly used measurement methods for HV in clinic situation. $^{18}$

\section{Statistical analysis}

A paired t-test was used to compare the differences in each variable within the two groups between the weight bearing and nonweight bearing conditions, and an independent t-test was used to compare the differences in weight bearing between the HV and 
Table 1. Comparison of the non-weight bearing and weight bearing conditions within groups $(\mathrm{N}=45)$

\begin{tabular}{|c|c|c|c|c|c|}
\hline & Group & Non-WT & WT & $t$ & $p$ \\
\hline \multirow[t]{2}{*}{ First MTPA $\left({ }^{\circ}\right)$} & Non-HV & $14.63 \pm 6.81$ & $10.34 \pm 3.30$ & 3.280 & $0.003^{*}$ \\
\hline & $\mathrm{HV}$ & $23.57 \pm 7.18$ & $18.88 \pm 3.06$ & 4.531 & $0.001^{*}$ \\
\hline \multirow[t]{2}{*}{ Second IMA $\left(^{\circ}\right)$} & Non-HV & $8.06 \pm 2.37$ & $9.58 \pm 2.38$ & -3.594 & $0.001^{*}$ \\
\hline & $\mathrm{HV}$ & $10.41 \pm 2.74$ & $11.28 \pm 2.61$ & -3.812 & $0.001^{*}$ \\
\hline \multirow[t]{2}{*}{ Distance of MSB (mm) } & Non-HV & $6.80 \pm 1.42$ & $5.92 \pm 1.63$ & -3.904 & $0.001^{*}$ \\
\hline & $\mathrm{HV}$ & $6.59 \pm 1.73$ & $5.15 \pm 2.04$ & -4.140 & $0.001^{*}$ \\
\hline
\end{tabular}

Values are expressed as mean \pm standard deviation (SD).

WT: weight bearing, HV: hallux valgus, MTPA: metatarsophalangeal joint angle, IMA: intermetatarsal angle, MSB: medial sesamoid bone. ${ }^{*} p<0.05$.

Table 2. Comparison on the difference of non-weight bearing and weight bearing conditions between groups $(\mathrm{N}=45)$

\begin{tabular}{lcccc}
\hline & Non-HV group & HV group & $\mathrm{t}$ & $\mathrm{p}$ \\
\hline First MTPA $\left(^{\circ}\right)$ & $-4.29 \pm 6.88$ & $-4.69 \pm 6.47$ & -0.294 & 0.804 \\
Second IMA $\left(^{\circ}\right)$ & $1.51 \pm 1.82$ & $0.87 \pm 1.61$ & -1.546 & 0.127 \\
Distance of MSB $(\mathrm{mm})$ & $-0.88 \pm 1.13$ & $-1.44 \pm 0.97$ & -2.218 & $0.030^{*}$ \\
\hline
\end{tabular}

Values are expressed as mean \pm standard deviation (SD).

HV: hallux valgus, MTPA: metatarsophalangeal joint angle, IMA: intermetatarsal angle, MSB: medial sesamoid bone.

${ }^{*} \mathrm{p}<0.05$.

non-HV groups. Statistical analysis was performed using the statistical package for the social sciences ver. 21 (SPSS, Chicago, IL, USA), and $\mathrm{p}<0.05$ was deemed to be statistically significant.

\section{RESULTS}

Both groups showed significant decrease in the distance of the MSB and the first MTP joint angle. A significant increase in the second IMA $(\mathrm{p}<0.05)$ was found in weight bearing condition compared to non-weight bearing (Table 1).

The difference in the distance of the MSB between weight bearing and non-weight bearing conditions was significantly greater in the HV group $(-1.44 \pm 0.97 \mathrm{~mm})$ than in the non-HV group $(-0.88 \pm$ $1.13 \mathrm{~mm})(\mathrm{p}<0.05)$. However, there were no significant differences between the two groups with respect to the first MTP joint angle (non-HV group $=-4.29 \pm 6.88^{\circ} ; \mathrm{HV}$ group $\left.=-4.69 \pm 6.47^{\circ}\right)$ and the second IMA (non-HV group $=1.51 \pm 1.82^{\circ} ; \mathrm{HV}$ group $=0.87 \pm 1.61^{\circ}$ ) $(\mathrm{p}>0.05)$ (Table 2).

\section{DISCUSSION}

Balance of the soft tissues in the great toe is important for normal biomechanics, and the sesamoid bones reflect this balance. ${ }^{12}$ In this study, the distance of the MSB from the midline of the first metatarsal bone was significantly shorter in both groups during weight bearing. The HV group had a significantly smaller MSB distance during weight bearing compared to the non-HV group. This reaffirmed that dislocation of the sesamoid bones occurs more frequently in the groups with $\mathrm{HV}^{3,17}$

Nayfa and Sorto ${ }^{19}$ reported a rapid increase in the HV angle after removal of MSB, mentioning that the MSB was important in balancing the force generated from the lateral sesamoid bone. For this reason, the distance of the MSB was measured to determine the location of the sesamoid bone.

For static measurement, Huang et $\mathrm{al}^{12}$ used lateral sesamoid bone measuring the vertical distance between the lateral edge of the lateral sesamoid bone and the axis of the second metatarsal bone. The authors determined the position of MSB based on where the MSB overlapped with the longitudinal axis of the first metatarsal as an index to assess their surgical results. It was classified into four grades: Grade 0 (no overlap with the sesamoid medial to the axis), Grade 1 (<50\% overlap), Grade 2 (>50\% overlap), and Grade 3 (no overlap with the sesamoid lateral to the axis). ${ }^{12}$ We attempted to use the same method, but accurate measurement was difficult to perform because the axis of the second metatarsal, which was the standard of measurement, shifted in the process of weight bearing positioning.

Reduction in the distance of the MSB from the midline of first metatarsal bone indicates dislocation of the MSB towards the lateral and plantar directions, which alters the function and lengths of the flexor hallucis longus and brevis muscles attached to the sesamoid bones. $^{6}$

In a previous electromyographic study, the muscle activity of flexor hallucis longus and brevis was decreased in flexion and increased in adduction in the HV group when compared with the 
normal group. ${ }^{20}$ Muscular weakness in the great toe was reported to undermine the normal propulsion mechanism during the toe-off stage of gait. ${ }^{20}$ These findings suggest that weight bearing affects the position of the sesamoid bones and altered position of sesamoid bones is associated with soft-tissue imbalance. Therefore, to measure the hallux valgus accurately and functionally, the position of the sesamoid bone should be assessed.

In the present study, both the HV and non-HV groups demonstrated a significant decrease in the first MTP joint angle and a significant increase in the second IMA in weight bearing, which were consistent with the results from previous studies. ${ }^{16,17}$ Although there were no significant differences between the two groups with respect to the first MTP joint angle and the second IMA, the variation amount of the mean value of the first MTP joint angle was large and that of the second IMA angle was small in the HV group compared to the non-HV group. These findings also indicate that the first MTP joint angle and the second IMA of HV group altered more than the non-HV group in weight bearing condition. The results of this study can be meaningful in determining the types of measurement posture and variables for more accurate clinical evaluations. Further research is needed to examine the kinematic changes in $\mathrm{HV}$ and the position of the sesamoid according to the degree of weight bearing in different age groups, including subjects with severe $\mathrm{HV}$.

The position of the sesamoid bones reflects the balance of the soft tissues in the great toe. A significant decrease was found in the distance of the MSB in the HV group when weight bearing and nonweight bearing conditions were compared. Although HV group showed decreased first MTP joint angle in weight bearing condition, MSB was found to be displaced towards the lateral and plantar directions. This could not only negatively affect the muscle balance of the great toe but to the normal gait. The results of this study present the necessity of considering these variables that occur in weight bearing when measuring the position of the MSB in HV patients.

\section{REFERENCES}

1. Yammine K. The sesamoids of the feet in humans: a systematic review and meta-analysis. Anat Sci Int. 2015;90(3):144-60.

2. Nwawka OK, Hayashi D, Diaz LE et al. Sesamoids and accessory ossicles of the foot: anatomical variability and related pathology. Insights Imag- ing. 2013;4(5):581-93.

3. Donatelli RA. The Biomechanics of the Foot and Ankle. 2nd ed. Philadelphia, FA Davis Company, 1996:26-31.

4. Maffulli N, Lepore L, Francobandiera C. Traumatic lesions of some accessory bones of the foot in sports activity. J Am Podiatr Med Assoc. 1990;80(2):86-90.

5. Shereff MJ, Bejjani FJ, Kummer FJ. Kinematics of the first metatarsophalangeal joint. J Bone Joint Surg Am. 1986;68(3):392-8.

6. Griffin LY. Essentials of musculoskeletal care. 3rd ed. Rosemont, American Academy of Orthopaedic Surgeons, 2005:819-21.

7. Kim MH, Koh EK, Jung DY. Analysis of kinematic motions of first metatarsophalangeal joint during electrical stimulation of abductor hallucis muscle in subjects with hallux valgus. J Kor Phys Ther. 2012;24(4):27681.

8. Choi GH, Park KY, Byun SJ et al. A study on angular correlation between hallux valgus and 1st MPJ dorsi-flexion according to work type and age of woman. J Kor Phys Ther. 2011;23(5):57-63.

9. Coughlin MJ, Jones CP. Hallux valgus: demographics, etiology, and radiographic assessment. Foot Ankle Int. 2007;28(7):759-77.

10. Saltzman CL, Aper RL, Brown TD. Anatomic determinants of first metatarsophalangeal flexion moments in hallux valgus. Clin Orthop Relat Res. 1997;(339):261-9.

11. Sanders AP, Snijders CJ, van Linge B. Medial deviation of the first metatarsal head as a result of flexion forces in hallux valgus. Foot Ankle. 1992;13(9):515-22.

12. Huang EH, Charlton TP, Ajayi S et al. Effect of various hallux valgus reconstruction on sesamoid location: a radiographic study. Foot Ankle Int. 2013;34(1):99-103.

13. Yun SJ, Kang MH, Kim MH. Difference of position change of sesamoid bones during active abduction exercise of great toe in subjects with hallux valgus. J Kor Phys Ther. 2015;27(2):85-8.

14. Kim MH, Yi CH, Weon JH et al. Effect of toe-spread-out exercise on hallux valgus angle and cross-sectional area of abductor hallucis muscle in subjects with hallux valgus. J Phys Ther Sci. 2015;27(4):1019-22.

15. Kang SY, Choung SD, Kim MH et al. Relationship between angle of metatarsophalangeal joint and abductor hallucis in hallux valgus. J Kor Phys Ther. 2014;26(2):56-61.

16. Fuhrmann RA, Layher F, Wetzel WD. Radiographic changes in forefoot geometry with weight bearing. Foot Ankle Int. 2003;24(4):326-31.

17. Tanaka Y, Takakura Y, Takaoka T et al. Radiographic analysis of hallux valgus in women on weight bearing and non-weight bearing. Clin Orthop Relat Res. 1997;(336):186-94.

18. Srivastava S, Chockalingam N, EI Fakhri T. Radiographic measurements of hallux angles: a review of current techniques. Foot (Edinb). 2010; 20(1):27-31.

19. Nayfa TM, Sorto LA. The incidence of hallux abductus following tibial sesamoidectomy. J Am Podiatry Assoc. 1982;72(12):617-20.

20. Arinci Incel N, Genç H, Erdem HR et al. Muscle imbalance in hallux valgus: an electromyographic study. Am J Phys Med Rehabil. 2003; 82(5):345-9.

21. Schubert DA. The role of the abductor hallucis in metatarsus primus varus associated with hallux valgus. J Am Podiatry Assoc. 1963;53:7524. 